\title{
The impact of large-scale, long-term optical surveys on pulsating star research
}

\author{
Igor Soszyński ${ }^{1, \star}$ \\ ${ }^{1}$ Warsaw University Observatory, Al. Ujazdowskie 4, 00-478 Warszawa, Poland
}

\begin{abstract}
The era of large-scale photometric variability surveys began a quarter of a century ago, when three microlensing projects - EROS, MACHO, and OGLE - started their operation. These surveys initiated a revolution in the field of variable stars and in the next years they inspired many new observational projects. Large-scale optical surveys multiplied the number of variable stars known in the Universe. The huge, homogeneous and complete catalogs of pulsating stars, such as Cepheids, RR Lyrae stars, or long-period variables, offer an unprecedented opportunity to calibrate and test the accuracy of various distance indicators, to trace the three-dimensional structure of the Milky Way and other galaxies, to discover exotic types of intrinsically variable stars, or to study previously unknown features and behaviors of pulsators. We present historical and recent findings on various types of pulsating stars obtained from the optical large-scale surveys, with particular emphasis on the OGLE project which currently offers the largest photometric database among surveys for stellar variability.
\end{abstract}

\section{Introduction}

Over the past quarter century, our knowledge of the pulsating stars has made a dramatic step forward and the lion's share of this progress came from large-scale optical surveys for stellar variability. The first such surveys were initiated in the early 1990s as a response to the suggestion of Bohdan Paczyński ([23]), who pointed out the possibility of using gravitational microlensing effects to detect massive compact objects in the Galactic halo. Paczyński predicted that photometric monitoring of a few million stars in the Magellanic Clouds over a time scale of two years should lead to a discovery of the first microlensing events (which in those days were hypothetical phenomena) and shed light on the nature of dark matter. This idea had a built-in "insurance policy" in case no gravitational microlensing events were detected. In the last sentence of his seminal paper Paczyński stated: "It is clear that the observational project is not simple, but one of its by-products, a systematic discovery of a large number of variable stars in a nearby galaxy, is attractive, even if no lensing events are discovered".

A few years later, three groups - EROS ([7]), MACHO ([1]), and OGLE ([59]) - started survey programs to search for microlensing events. The EROS (Expérience de Recherche d'Objets Sombres) experiment was a French collaboration between astronomers and particle physicists. This project was conducted from 1990 to 2003 and it was divided into two phases. In the first phase (EROS-1), it used two telescopes located at the ESO La Silla Observatory in Chile: a 1-meter Schmidt telescope and a

\footnotetext{
^soszynsk@astrouw.edu.pl
} 
Table 1. Parts of the OGLE Collection of Variable Stars published so far.

\begin{tabular}{|c|c|c|c|}
\hline Type of variable stars & Environments $^{1}$ & Number of stars & References \\
\hline Classical Cepheids & LMC, SMC, GB, GD & 9587 & {$[28],[48],[53]$} \\
\hline Type II Cepheids & LMC, SMC, GB & 603 & {$[41],[45],[48]$} \\
\hline Anomalous Cepheids & LMC, SMC & 250 & [52] \\
\hline RR Lyrae stars & LMC, SMC, GB, GD & 83753 & {$[28],[51],[54]$} \\
\hline Long-Period Variables & LMC, SMC, GB & 343785 & [43], [47], [49] \\
\hline$\delta$ Scuti stars & LMC, GD & 2844 & {$[28],[35]$} \\
\hline $\begin{array}{l}\text { Eclipsing and ellipsoidal } \\
\text { binary systems }\end{array}$ & LMC, SMC, GB, GD & 510792 & {$[24],[27],[55]$} \\
\hline Total & & 951614 & \\
\hline
\end{tabular}

0.4-meter telescope equipped with a CCD camera. In the second stage of the survey (EROS-2), the 1-meter Marly telescope at the same observatory was used. EROS monitored the Large (LMC) and Small (SMC) Magellanic Clouds, the center of the Milky Way, and four directions in the Galactic disk. The total number of sources in the EROS-2 database is about 87 million.

The MACHO (Massive Compact Halo Object) project was active for 8 years (1992-1999) and monitored 62 million stars in the Magellanic Clouds and the bulge of the Milky Way. This survey has had full-time use of the 1.27-meter telescope at Mount Stromlo Observatory, Australia, which was a rebuilt historical telescope known as the Great Melbourne Telescope from 1868. In the nineteenth century it was the second largest telescope in the world and the largest in the southern hemisphere. MACHO simultaneously observed in two colors, using eight $2048 \times 2048$ pixel CCDs.

The OGLE (Optical Gravitational Lensing Experiment) project is the only active one of these pioneering microlensing surveys which started in early nineties. Within a quarter of a century, OGLE has been evolving, gradually increasing its observing capabilities. Currently, in its fourth phase (OGLEIV, [62]), the survey daily monitors over a billion objects in the densest stellar regions of the sky: central regions of the Milky Way, the Galactic disk, and the Magellanic Clouds. To date, the project has collected about one trillion $\left(10^{12}\right)$ individual photometric measurements, making OGLE the world's largest survey aimed at searching for variability in the sky. The project uses a dedicated 1.3-meter Warsaw telescope at Las Campanas Observatory, Chile, equipped with a 32-chip mosaic CCD camera. The observations are conducted using the standard $V$ - and $I$-band filters, which ensures direct use of the OGLE photometry in many astrophysical applications or comparison with existing older data.

During its long history the OGLE project contributed significantly to many fields of observational astrophysics: gravitational lensing and microlensing, extrasolar planets, cosmic distance scale, stellar clusters, interstellar extinction, Kuiper Belt objects, active galactic nuclei, etc. Variable stars occupy a special position among the most important achievements of the survey. OGLE is an unquestionable world leader in this field. The OGLE Collection of Variable Stars contains presently nearly one million objects of various types and this is the largest set of variable stars ever obtained by any astronomical project. Table 1 summarizes the number of variable stars published by OGLE so far.

In this paper, we present the most important results on pulsating stars obtained by the large-scale optical surveys. Our goal is to show the incredible progress that has been made in this field in the last 25 years. In the next sections we present respectively classical Cepheids, RR Lyrae stars, type II Cepheids, anomalous Cepheids, long-period variables, and other pulsating stars. 


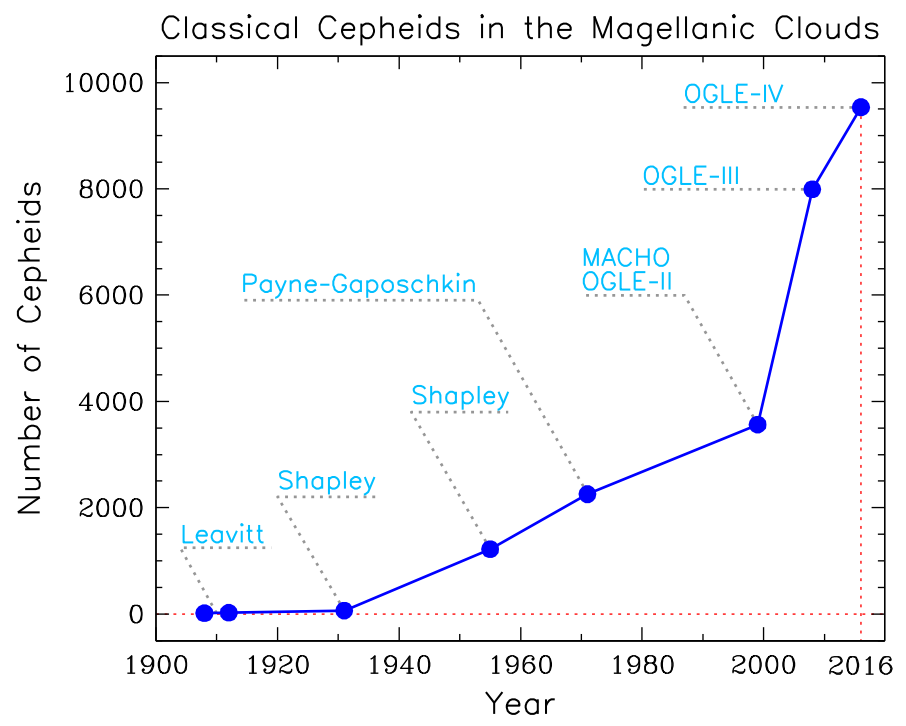

Figure 1. Number of known classical Cepheids in the Magellanic Clouds from the beginning of the twentieth century. References: [18], [19], [36], [37], [25], [26], [5], [60], [61], [40], [44], [53].

\section{Classical Cepheids}

Classical Cepheids are one of the most important distance indicators and tracers of the young stellar population. They are population I, relatively massive $\left(M>4 M_{\odot}\right)$ giant stars obeying the famous period-luminosity (PL) relationship discovered in the Magellanic Clouds by Henrietta Leavitt in 1908 ([18]). Figure 1 shows how many classical Cepheids in the Magellanic Clouds have been known from the beginning of the twentieth century, starting from 16 Cepheids $^{1}$ discovered by Leavitt ([18]), through several review and catalog papers summarizing the number of Cepheids known in those days $([25,26,36,37])$, the first catalogs of Cepheids in the Magellanic Clouds released by the MACHO and OGLE-II projects in $1999([5,60,61])$, ending with a real breakthrough that was made in recent years by OGLE-III $([40,44])$ and OGLE-IV projects ([53]). Currently, we almost reached the maximum value of this distribution. The total number of classical Cepheids in both Clouds is about 10000 .

\subsection{Period-luminosity relations}

The progress that has been made in this field is well illustrated in Figure 2. We present here four PL diagrams for classical Cepheids in the Magellanic Clouds. From left to right and from top to bottom we present here the historically first PL diagram plotted in 1912 by Leavitt ([19]) for 25 Cepheids in the SMC (two relationships result from the fact that each Cepheid is represented by two points corresponding to the minimum and maximum light), the period-Wesenheit index diagram obtained by Barry Madore in 1982 ([20]) containing all LMC Cepheids with high-quality photoelectric observations published in those days, the PL diagram with 1800 LMC Cepheids observed by the MACHO team ([5]), and the newest PL diagram with 4620 LMC classical Cepheids cataloged by the OGLE

\footnotetext{
${ }^{1}$ Actually, Leavitt ([18]) discovered a larger number of Cepheids, but in 1908 the classification was firm only for 16 of them for which periods were determined.
} 

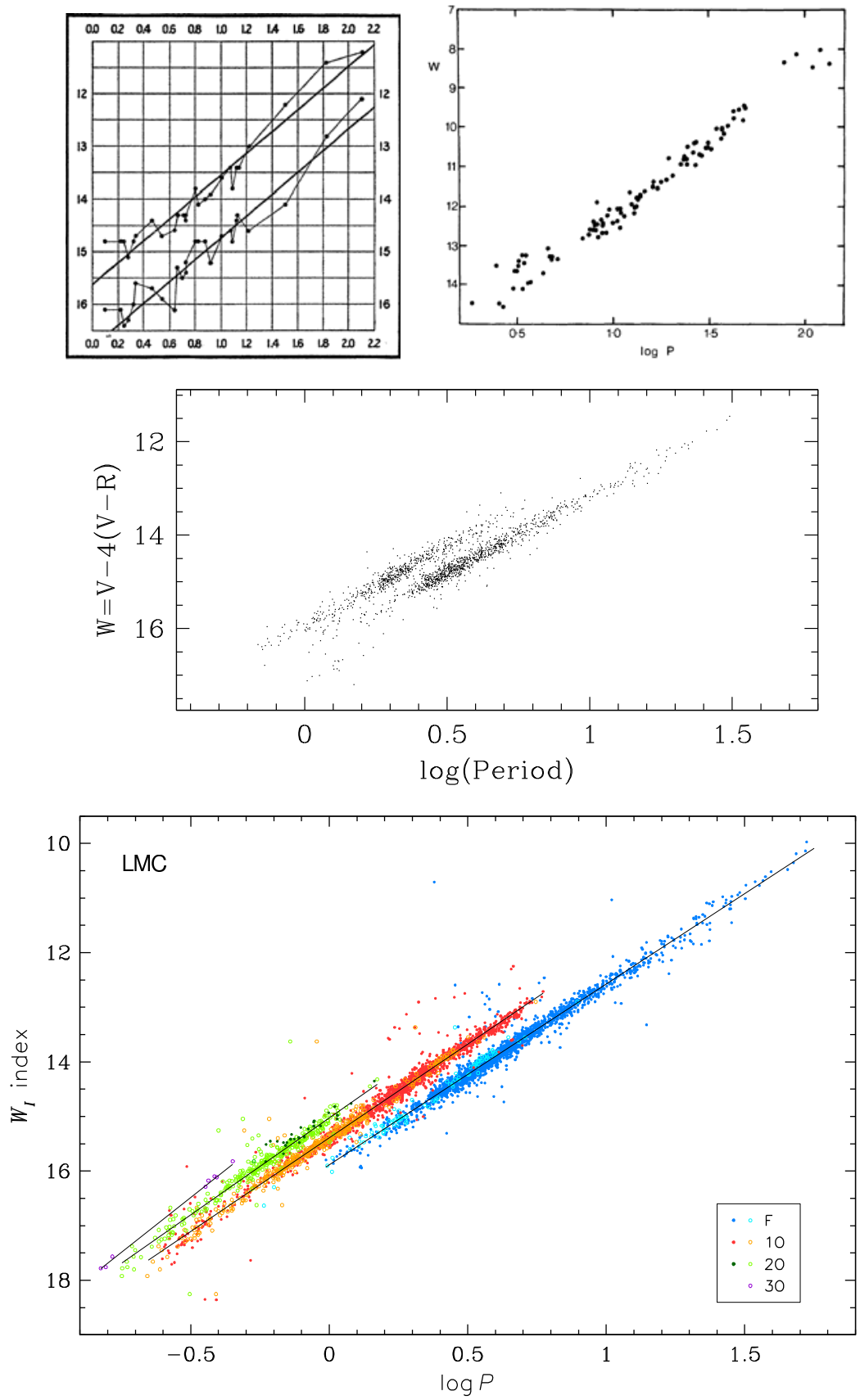

Figure 2. Period-luminosity diagrams for classical Cepheids in the Magellanic Clouds. From left to right and from top to bottom: the first PL diagram plotted in 1912 by Henrietta Leavitt for 25 Cepheids ([19]), the period-Wesenheit index plot obtained in 1982 by Barry Madore for 96 Cepheids with high-quality photoelectric observations ([20]), the PL diagram with 1800 Cepheids identified by the MACHO survey in 1999 ([5]), and the most recent PL diagram containing 4620 classical Cepheids in the LMC detected by the OGLE project ([53]). 
survey ([53]). Note that only the microlensing surveys showed the two PL sequences of Cepheids for those pulsating in the fundamental mode and the first overtone mode. In the most recent OGLE diagram, we can distinguish as many as four PL relations populated by the fundamental, first overtone, second overtone and third overtone Cepheids.

The Magellanic Clouds played in the past and still play a crucial role in the studies and calibrations of the PL relations of Cepheids and other pulsating stars. There is still an open question for the linearity and universality of the PL relations and its dependence on the chemical composition of the stars. The extraordinary large number of Cepheids discovered by the microlensing surveys allowed many authors to test the linearity of the PL relations with unprecedented precision. The first nonlinearity around the periods of 2.5 days in the SMC Cepheids were noticed on the basis of the data published by the EROS survey ([8]). Then, the studies of the PL and period-color relations for Cepheids have been presented in countless papers. Cepheids in the LMC and SMC pulsating in the fundamental and first-overtone modes, using various pass-band from visual to infrared, have been thoroughly tested.

These studies are essential for using Cepheids as distance indicators, but they are also used to study the three-dimensional structure of the Magellanic System. Among many papers on this topic, we would like to draw attention to a recent work by Jacyszyn-Dobrzeniecka et al. ([17]), who used the most complete collection of classical Cepheids from the OGLE-IV project to describe the structures of both Clouds. They also studied the young stellar population in the Magellanic Bridge, since several classical Cepheids were recently discovered in this region ([53]).

\subsection{Multi-mode Cepheids}

Multi-mode Cepheids and other classical pulsators deserve special attention, since they show well the progress that has been made thanks to the large-scale surveys. In the pre-microlensing era the picture was simple - only about ten double-mode Cepheids had been known. Most of them pulsated simultaneously in the fundamental- and first-overtone modes $(\mathrm{F} / 1 \mathrm{O})$ and only one classical Cepheid CO Aur - had the first and second overtones $(1 \mathrm{O} / 2 \mathrm{O})$ excited, although the excitation of the second overtone in this star was disputed.

The revolution in this field began in 1995 when the MACHO team announced the discovery of 45 double-mode Cepheids in the LMC ([2]) - four times more than had been known before. The observations from the microlensing survey gave the first unambiguous evidence that the second overtone can be excited in Cepheids. The most recent collection of Cepheids published by OGLE ([53]) contains more than 700 double-mode Cepheids. The Petersen diagram (period ratio versus the longer period) for the Magellanic Clouds Cepheids is shown in Figure 3. Here, we also plot two double-mode classical Cepheids belonging to previously unknown classes: oscillating simultaneously in the first and third overtones (1O/3O) and second and third overtones (2O/3O). Triple-mode Cepheids are marked with triangles in Figure 3. About ten such unique pulsators are currently known and all of them have been discovered in the databases of the large-scale surveys. Finally, the non-radial modes in classical Cepheids manifest themselves in the form of three sequences in the Petersen diagram around the period ratios between 0.60 to 0.65 (empty circles in Fig. 3). Recently, Dziembowski ([9]) proposed an interesting theoretical interpretation of these modes. More information about the "revolution" in the Petersen diagram can be found in the papers by Radosław Smolec and Henryka Netzel in these proceedings.

\subsection{Cepheids in eclipsing binary systems}

Classical Cepheids which are members of eclipsing binary systems are very rare objects. No such star is currently known in the Milky Way. All of the known objects of this type belong to the Magellanic 


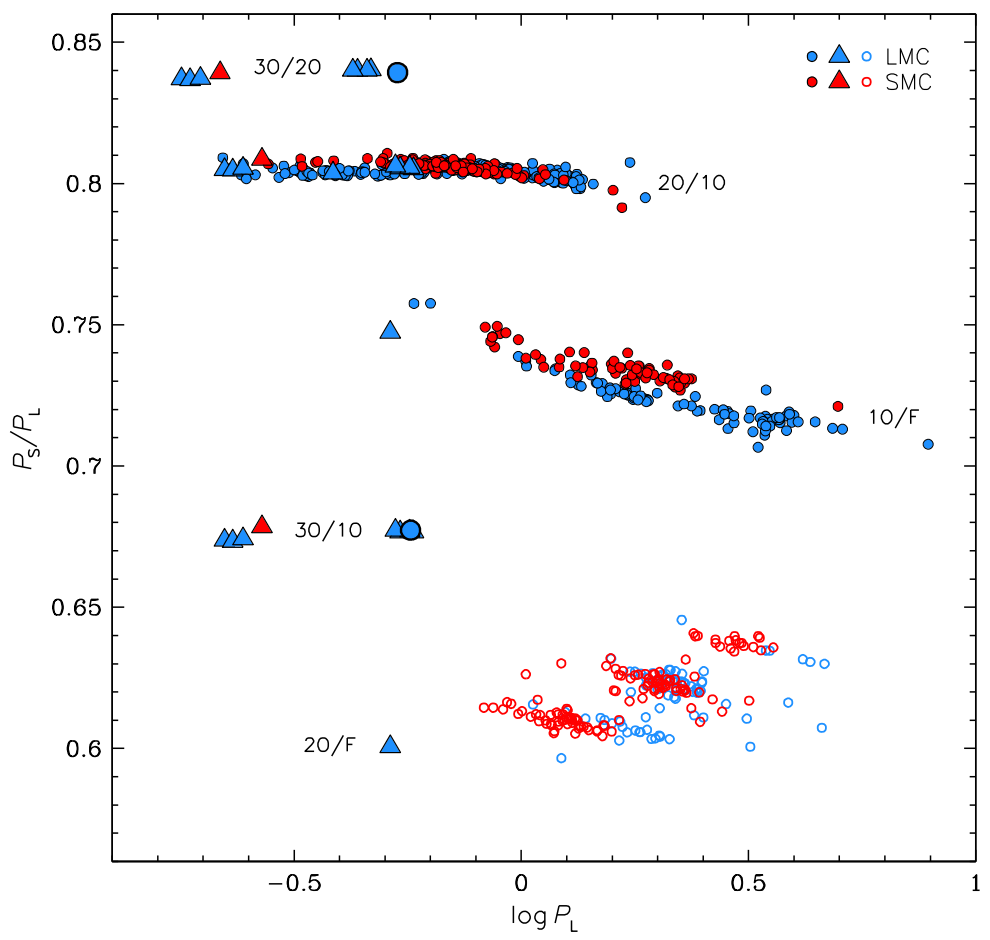

Figure 3. Petersen diagram for multi-mode classical Cepheids in the Magellanic Clouds. Solid dots show doublemode variables, triangles mark triple-mode Cepheids (three points per star) and empty circles represent Cepheids with non-radial modes excited. Blue and red symbols indicate stars in the LMC and SMC, respectively.

Clouds and all of them were discovered by the OGLE survey ([63]). Eclipsing binary systems hosting Cepheids have several interesting astrophysical applications. First, photometric and spectroscopic analyses of such objects may provide masses and radii of pulsating stars with great accuracy. It is particularly important in the context of the longstanding mass discrepancy problem for classical Cepheids. Second, detached double-lined eclipsing binary systems containing Cepheids are promising tools to calibrate the Cepheid PL relation, since a careful analysis of such systems may provide their accurate distances. Third, it is possible to precisely determine the so called $p$-factor - the crucial parameter in the Baade-Wesselink method of distance determinations to Cepheids. The Araucaria project ([11]) specializes in the in-depth analysis of pulsating stars in eclipsing binary systems (e.g., $[12,31,34])$.

\subsection{Cepheids in the Milky Way}

Besides the Magellanic Clouds, a number of classical Cepheids were identified by the OGLE team in the central regions of our Galaxy ([48]). Using this sample, Feast et al. ([10]) found an unexpected young stellar component of the flared outer disk located behind the Galactic center. This discovery clearly showed that the structure of the Galaxy is far from being fully understood. The cause behind the flaring of the disk remains uncertain, but one possible explanation is the presence of dark matter. 


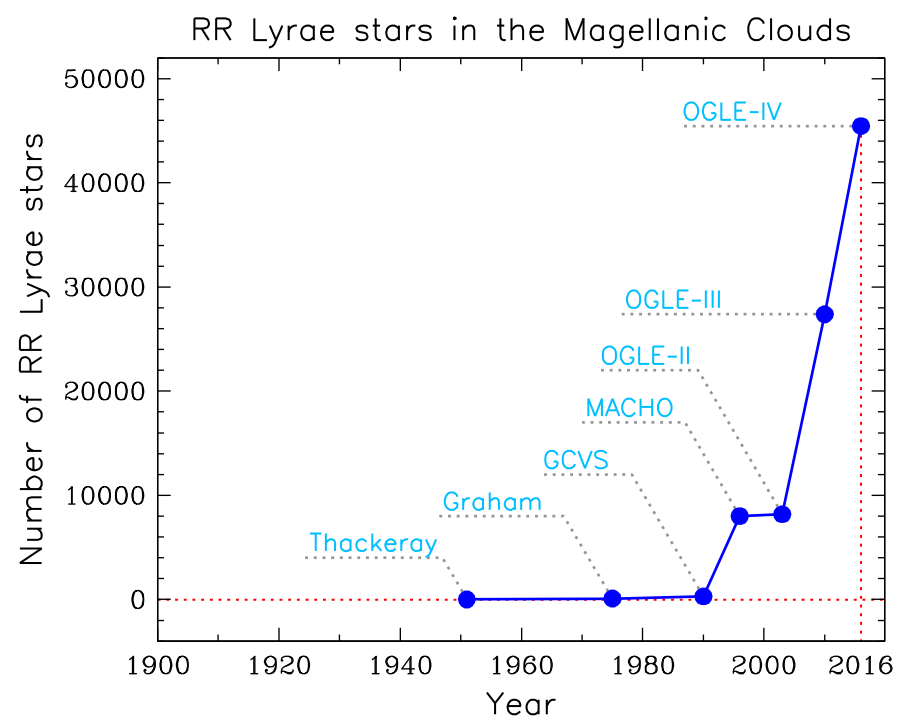

Figure 4. Number of known RR Lyrae stars in the Magellanic Clouds from the beginning of the twentieth century. References: [57], [14], [6], [3], [42], [46], [54].

Also the collection of known classical Cepheids in the Milky Way will be soon significantly extended thanks to a new subproject of the OGLE project, the OGLE Galaxy Variability Survey - a long-term photometric survey for stellar variability in the Galactic disk and in the outer regions of the Galactic bulge. This project covers nearly $2 / 3$ of the Galactic plane with Galactic longitudes from $-170^{\circ}$ to $+60^{\circ}$ and latitudes from $-3^{\circ}$ to $+3^{\circ}$ in the disk and from $-15^{\circ}$ to $+15^{\circ}$ in the bulge region. The total observed area is about 2000 square degrees covered by about 1200 individual pointings. For the preliminary results of the search for Cepheids and RR Lyrae stars see the paper by Andrzej Udalski in these proceedings.

\section{RR Lyrae stars}

RR Lyrae stars are indispensable tracers of, and distance indicators for, the old stellar population in our own and nearby galaxies. These radially pulsating horizontal branch stars populate galactic halos, thick disks and globular clusters. RR Lyr variables are numerous, present in various stellar environments, and are relatively easy to detect, when precise, well covered light curves are available.

Figure 4 illustrates how the number of known RR Lyr stars in the Magellanic System has increased over the years. The first RR Lyr variables in the Magellanic Clouds were identified by A. David Thackeray in 1951 ([57]), but 40 years later only about 300 RR Lyr stars were known in both galaxies ([6]), mostly in the fields around globular clusters. The breakthrough came with the large-scale surveys, in particular the OGLE project, which increased the number of identified RR Lyr stars in the Magellanic Clouds to over 45000 ([54]). Huge samples of RR Lyr stars were also found in our Galaxy: over 38000 objects were discovered in the bulge by the OGLE team ([51]), about 22000 stars in the Milky Way halo were identified by the Catalina survey ([58]), and an impressive number of $150000 \mathrm{RR}$ Lyr candidates were selected in the database of the Pan-STARRS survey ([16]). However, the purity and 
completeness of the latter sample is significantly reduced due to a limited number of observing points collected to date by the Pan-STARRS project.

These numerous sets of RR Lyrae stars have proven to be a powerful probe of the Galactic structure. Pietrukowicz et al. ([30]) used the OGLE RR Lyr variables to show that the old stellar population traces the barred structure of the Galactic bulge. In the Galactic halo several overdensities have been discovered $([16,58])$ with the most pronounced being the Sagittarius Stream, which consists of tidally stripped stars from the Sagittarius Dwarf Galaxy. It is worth mentioning that the central regions of the Sagittarius Dwarf Galaxy were recently explored by Hamanowicz et al. ([15]) based on RR Lyr stars published by OGLE.

Moreover, the collection consisting of tens of thousands of RR Lyr variables with long-term, well covered light curves is an inexhaustible source of unique pulsators exhibiting exotic behaviors. Let us mention the discovery of a new class of double-mode RR Lyr variables (anomalous RRd stars, [56]), the detection of non-radial modes in RR Lyr stars (e.g., [22]), the first observations of the Blazhko effect in RRd stars ([38]), RR Lyr stars switching their pulsation modes ([50, 51]), and a candidate for an RR Lyr star in an eclipsing binary system (OGLE-BLG-RRLYR-02792), which turned out to be the first known representative of a new type of pulsating stars mimicking classical RR Lyr stars ([32]).

\section{Type II Cepheids}

Type II Cepheids belong to the same population as RR Lyr stars, but are much less numerous. There are several hundreds of type II Cepheids known in the Galaxy and Magellanic Clouds. This class of variable stars is traditionally divided into three groups, each one at a different evolutionary stage: BL Her stars with periods between 1 and 4 days, W Vir stars with periods in the range 4-20 days and RV Tau stars with periods above 20 days and characteristic alternating deep and shallow minima.

Observations obtained by the large-scale programs, in particular MACHO and OGLE, led to very important discoveries about type II Cepheids. Alcock et al. ([4]) found the first certain RV Tau stars in the LMC and derived their absolute magnitudes for the first time confirming that they are luminous, post-AGB stars. This discovery showed that RV Tau stars obey a PL relation which is an extension of the PL relation delineated by other type II Cepheids - BL Her and W Vir stars.

One of the most important achievements of the OGLE project was the discovery of the fourth subclass of type II Cepheids, which was called peculiar W Vir stars ([41]). These pulsating variables exhibit distinct features of their light curves and are on average bluer and brighter than regular W Vir stars. Moreover, a large fraction of peculiar W Vir stars show eclipsing or ellipsoidal modulation superimposed on the pulsation light curves, which suggests that all these stars are members of binary systems.

\section{Anomalous Cepheids}

Until the discovery of the first bona fide anomalous Cepheids in the Magellanic Clouds by the OGLE team $([41,52])$, the vast majority of this kind of pulsators was known in dwarf galaxies. The sample of 250 anomalous Cepheids detected by OGLE in the LMC and SMC multiplied the total number of such stars known in the Universe.

The origin of anomalous Cepheids is still a subject of debate. There is a consensus that they are metal-deficient core-helium-burning stars with masses $1-2 M_{\odot}$. They pulsate in the fundamental mode or in the first-overtone mode and their PL relations are located between the relations for classical and type II Cepheids. There are two leading hypotheses for the origin of anomalous Cepheids: that they are intermediate-age stars (1-6 Gyr old) with exceptionally low metallicity or that they are coalesced old (>10 Gyr) binary stars. 
An analysis of the spatial distribution of anomalous Cepheids in the Magellanic Clouds gives a hint for their origin ([52]). Their distribution is much more similar to the old stellar population represented by RR Lyr stars than to the young classical Cepheids. However, some anomalous Cepheids in the LMC seem to trace spiral arms, which makes them similar to the younger stars. The conclusion is that anomalous Cepheids are probably a mixture of old and intermediate-age stars.

The large and homogeneous sample of anomalous Cepheids discovered by OGLE in the Magellanic Clouds was also used to demonstrate that the coefficients derived from the Fourier light curve decomposition are unambiguous discriminators between classical and anomalous Cepheids. This finding enabled Soszyński et al. ([52]) to discover four Galactic anomalous Cepheids in the foreground of the Magellanic Clouds - the first fundamental-mode stars of that type in the field (outside clusters) of the Milky Way. This discovery showed that anomalous Cepheids must be quite numerous in the Galactic halo.

\section{Long-period variables}

Long-period variables (LPVs), or in other words pulsating red giants, constitute a very numerous group of variable stars. They are bright, some of them (Miras) exhibit very large amplitudes, but they belong to the least studied types of pulsating stars. The reason for this lies in their long periods, from weeks to years, and usually complex nature of their light curves. The study of LPVs benefited the most from the long-term monitoring by the microlensing surveys.

Figure 5 illustrates the history of discovering the PL relations for LPVs. The first such relation was noticed in 1981 by Glass and Lloyd-Evans ([13]) for Miras. Then, the second PL sequence was found in 1996 by Wood and Sebo ([64]) for semiregular variables. Other sequences in the PL plane were discovered on the basis of the data collected by the large-scale surveys. Wood ([65]) used the MACHO database to find five PL relations obeyed by LPVs, while the OGLE data allowed us to distinguish more than ten sequences ([39]). Most of these relations are already linked to particular radial and non-radial modes of pulsation ([21]); however, sequence D, populated by the so called Long Secondary Periods, still does not have a satisfactory explanation.

\section{Blue pulsating stars}

The variability surveys have strongly contributed to our knowledge about pulsating stars on the blue side of the HR diagram. An achievement of the ASAS project (All Sky Automated Survey, one of the first whole sky surveys conducted with fully automated small telescopes) may be given as example. Pigulski \& Pojmański ([33]) reported the discovery of nearly 300 new $\beta$ Cep stars in the ASAS survey - three times more than were known before. Such a large sample opened the possibility to perform a series of statistical analyses of $\beta$ Cep stars and to compare the results with pulsation theory.

Another very fresh discovery comes from the OGLE project. OGLE-GD-DSCT-0058 is a star in the Galactic disk which was initially classified as a $\delta$ Sct star ([28]). Its period is only 28 minutes, it has a large amplitude, and its light curve morphology is typical for fundamental-mode pulsating stars. Surprisingly, the spectrum obtained by Pietrukowicz et al. ([29]) showed that the effective temperature of this star is $30000 \mathrm{~K}$, which corresponds to spectral type 09 - so this object cannot be a $\delta$ Sct star. The surface gravity places this star between main-sequence stars and subdwarf OB stars. OGLE-GDDSCT-0058 is probably the first representative of a new type of pulsating stars, tentatively called Blue Large-Amplitude Pulsators (BLAPs). A careful search in the OGLE photometric databases resulted in the discovery of more than a dozen additional BLAPs in the Galactic bulge. 

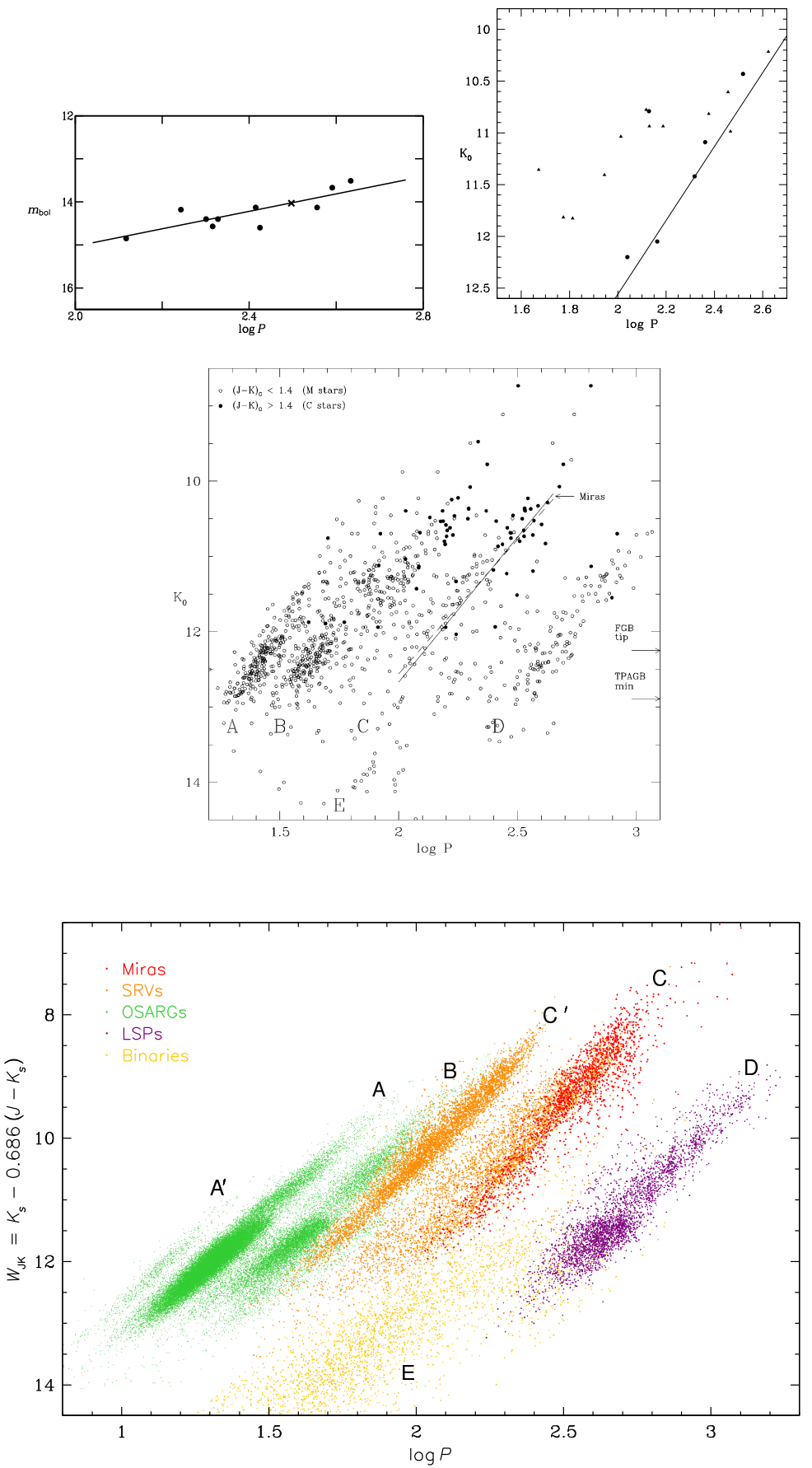

Figure 5. Period-luminosity diagrams for long-period variables in the LMC. From left to right and from top to bottom: the near-infrared PL diagram obtained by Glass and Lloyd Evans in 1981 for 11 Miras ([13]), two PL relations (for Miras and semiregular variables) discovered by Wood \& Sebo in 1996 ([64]), the PL diagram plotted by Peter Wood in 1999 on the basis of the MACHO observations ([65]), and the modern PL diagram for LPVs from the OGLE Collection of Variable Stars ([43]). 


\section{Conclusions}

The large-scale optical observational projects have radically changed the way we understand pulsating stars. Hundreds of thousands of new variable objects have been identified and cataloged on the basis of the photometry collected in the course of these projects. Studies of such huge samples of variable stars have at least three advantages. First, it is possible to examine in detail the statistical properties of pulsators (e.g., PL relations). Second, in the large sets of variable stars of a given type it is easier to find objects of very rare subtypes, or even those previously unknown. Third, complete and homogeneous samples of pulsators can be used to study the structure and evolution of our and other galaxies.

Large-scale photometric surveys practically demonstrated that it is feasible to monitor billions of stars per night, making up to $10^{11}$ photometric measurements per year. Long-term, well covered light curves are ideal to probe the photometric behavior of pulsating stars for timescales from minutes to years. The technology is still relatively cheap. The question arises, what is the future of large-scale sky surveys? The existing projects cover the densest stellar regions of the sky: the Galactic bulge and disk and the Magellanic Clouds. Outside these regions the number of stars is much smaller, so further expanding the sky coverage in existing projects will not significantly increase the number of monitored stars. It seems that further development will go toward deeper photometry (LSST), higher time resolution, and broader spectral coverage.

Acknowledgments: This work has been supported by the Polish National Science Centre grant MAESTRO no. 2014/14/A/ST9/00121.

\section{References}

[1] Alcock, C., Akerlof, C. W., Allsman, R. A., et al., Nature, 365, 621 (1993)

[2] Alcock, C., Allsman, R. A., Axelrod, T. S., et al., AJ, 109, 1654 (1995)

[3] Alcock, C., Allsman, R. A., Axelrod, T. S., et al., AJ, 111, 1146 (1996)

[4] Alcock, C., Allsman, R. A., Alves, D. R., et al., AJ, 115, 1921 (1998)

[5] Alcock, C., Allsman, R. A., Alves, D. R., et al., AJ, 117, 920 (1999)

[6] Artyukhina, N. M., Durlevich, O. V., Frolov, M. S., et al., General Catalogue of Variable Stars, 4rd ed., vol. V. Extragalactic Variable Stars, "Kosmosinform", Moscow (1995)

[7] Aubourg, E., Bareyre, P., Bréhin, S., et al., Nature, 365, 623 (1993)

[8] Bauer, F., Afonso, C., Albert, J. N., et al., A\&A, 348, 175 (1999)

[9] Dziembowski, W. A., Communications of the Konkoly Observatory Hungary, 105, 23 (2016)

[10] Feast, M. W., Menzies, J. W., Matsunaga, N., \& Whitelock, P. A., Nature, 509, 342 (2014)

[11] Gieren, W., Pietrzyński, G., Bresolin, F., et al., The Messenger, 121, 23 (2005)

[12] Gieren, W., Pilecki, B., Pietrzyński, G., et al., ApJ, 815, 28 (2015)

[13] Glass, I. S., \& Evans, T. L., Nature, 291, 303 (1981)

[14] Graham, J. A., PASP, 87, 641 (1975)

[15] Hamanowicz, A., Pietrukowicz, P., Udalski, A., et al., Acta Astron., 66, 197 (2016)

[16] Hernitschek, N., Schlafly, E. F., Sesar, B., et al., ApJ, 817, 73 (2016)

[17] Jacyszyn-Dobrzeniecka, A. M., Skowron, D. M., Mróz, P., et al., Acta Astron., 66, 149 (2016)

[18] Leavitt, H. S., Annals of Harvard College Observatory, 60, 87 (1908)

[19] Leavitt, H. S., \& Pickering, E. C., Harvard College Observatory Circular, 173, 1 (1912)

[20] Madore, B. F., ApJ, 253, 575 (1982)

[21] Mosser, B., Dziembowski, W. A., Belkacem, K., et al., A\&A, 559, A137 (2013) 
[22] Netzel, H., Smolec, R., \& Moskalik, P., MNRAS, 447, 1173 (2015)

[23] Paczyński, B., ApJ, 304, 1 (1986)

[24] Pawlak, M., Soszyński, I., Udalski, A., et al., Acta Astron., 66, 421 (2016)

[25] Payne-Gaposchkin, C., \& Gaposchkin, S., Smithsonian Contrib. Astrophys., 9, 1 (1966)

[26] Payne-Gaposchkin, C. H., Smithsonian Contrib. Astrophys., 13 (1971)

[27] Pietrukowicz, P., Mróz, P., Soszyński, I., et al., Acta Astron., 63, 115 (2013a)

[28] Pietrukowicz, P., Dziembowski, W. A., Mróz, P., et al., Acta Astron., 63, 379 (2013b)

[29] Pietrukowicz, P., Latour, M., Angeloni, R., et al., Acta Astron., 65, 63 (2015a)

[30] Pietrukowicz, P., Kozłowski, S., Skowron, J., et al., ApJ, 811, 113 (2015b)

[31] Pietrzyński, G., Thompson, I. B., Gieren, W., et al., Nature, 468, 542 (2010)

[32] Pietrzyński, G., Thompson, I. B., Gieren, W., et al., Nature, 484, 75 (2012)

[33] Pigulski, A., \& Pojmański, G., AIPC, 1170, 351 (2009)

[34] Pilecki, B., Graczyk, D., Gieren, W., et al., ApJ, 806, 29 (2015)

[35] Poleski, R., Soszyński, I., Udalski, A., et al., Acta Astron., 60, 1 (2010)

[36] Shapley, H., Harvard College Observatory Bulletin, 883, 16 (1931)

[37] Shapley, H., \& McKibben Nail, V., Proc. Nat. Acad. Sci., 41, 829 (1955)

[38] Smolec, R., Soszyński, I., Udalski, A., et al., MNRAS, 447, 3756 (2015)

[39] Soszyński, I., Dziembowski, W. A., Udalski, A., et al., Acta Astron., 57, 201 (2007)

[40] Soszyński, I., Poleski, R., Udalski, A., et al., Acta Astron., 58, 163 (2008a)

[41] Soszyński, I., Udalski, A., Szymański, M. K., et al., Acta Astron., 58, 293 (2008b)

[42] Soszyński, I., Udalski, A., Szymański, M. K., et al., Acta Astron., 59, 1 (2009a)

[43] Soszyński, I., Udalski, A., Szymański, M. K., et al., Acta Astron., 59, 239 (2009b)

[44] Soszyński, I., Poleski, R., Udalski, A., et al., Acta Astron., 60, 17 (2010a)

[45] Soszyński, I., Udalski, A., Szymański, M. K., et al., Acta Astron., 60, 91 (2010b)

[46] Soszyński, I., Udalski, A., Szymański, M. K., et al., Acta Astron., 60, 165 (2010c)

[47] Soszyński, I., Udalski, A., Szymański, M. K., et al., Acta Astron., 61, 217 (2011a)

[48] Soszyński, I., Udalski, A., Pietrukowicz, P., et al., Acta Astron., 61, 285 (2011b)

[49] Soszyński, I., Udalski, A., Szymański, M. K., et al., Acta Astron., 63, 21 (2013)

[50] Soszyński, I., Dziembowski, W. A., Udalski, A., et al., Acta Astron., 64, 1 (2014a)

[51] Soszyński, I., Udalski, A., Szymański, M. K., et al., Acta Astron., 64, 177 (2014b)

[52] Soszyński, I., Udalski, A., Szymański, M. K., et al., Acta Astron., 65, 233 (2015a)

[53] Soszyński, I., Udalski, A., Szymański, M. K., et al., Acta Astron., 65, 297 (2015b)

[54] Soszyński, I., Udalski, A., Szymański, M. K., et al., Acta Astron., 66, 131 (2016a)

[55] Soszyński, I., Pawlak, M., Pietrukowicz, P., et al., Acta Astron., 66, 405 (2016b)

[56] Soszyński, I., Smolec, R., Dziembowski, W. A., et al., MNRAS, 463, 1332 (2016c)

[57] Thackeray, A. D., \& Wesselink, A. J., Nature, 171, 693 (1953)

[58] Torrealba, G., Catelan, M., Drake, A. J., et al., MNRAS, 446, 2251 (2015)

[59] Udalski, A., Szymański, M., Kałużny, J., Kubiak, M., \& Mateo, M., Acta Astron., 42, 253 (1992)

[60] Udalski, A., Soszynski, I., Szymanski, M., et al., Acta Astron., 49, 223 (1999a)

[61] Udalski, A., Soszynski, I., Szymanski, M., et al., Acta Astron., 49, 437 (1999b)

[62] Udalski, A., Szymański, M. K., \& Szymański, G., Acta Astron., 65, 1 (2015a)

[63] Udalski, A., Soszyński, I., Szymański, M. K., et al., Acta Astron., 65, 341 (2015b)

[64] Wood, P. R., \& Sebo, K. M., MNRAS, 282, 958 (1996)

[65] Wood, P. R., PASA, 17, 18 (2000) 